\title{
Clinicopathological profile of neuroendocrine tumors of gastrointestinal tract
}

\author{
Jeya Shambavi J. ${ }^{1}$, Narmadhar R. ${ }^{2}$, Rajalakshmi V. ${ }^{3}$, Kalaivani Selvi S. ${ }^{4, *}$ \\ $\mathbf{1 , 2 , 4}^{\mathbf{1}}$ Assistant Professor, ${ }^{\mathbf{3}}$ Professor, ${ }^{\mathbf{1}}$ Aarupadai Veedu Medical College, Kirumampakkam, Puducherry, ${ }^{\mathbf{2}}$ Madras Medical College, \\ Chennai, Tamil Nadu, ${ }^{3}$ ESIC Medical College \& PGIMSR, Chennai, Tamil Nadu, ${ }^{4}$ Sri Manakula Vinayagar Medical College and \\ Hospital, Puducherry, India
}

*Corresponding Author:

Email: klvnselvi@yahoo.co.in

\begin{abstract}
Introduction: Neuroendocrine neoplasms are derived predominately from enterochromaffin or Kulchitsky's cells. The estimated prevalence of neuroendocrine tumors (NET) is 1 to 2 cases per 100,000 people, of which gastrointestinal tract (GIT) is the most common site. And being a rare tumor, it is less studied

Aim of the Study: To study the clinicopathological profile of Neuroendocrine tumors of GIT.

Materials and Methods: All specimens of neuroendocrine tumors received from the Department of Surgery and Department of digestive health diseases during the period from September 2008 to September 2012 were included. Clinical details were collected from the medical records in all cases. The tumors were classified based on WHO classification 2010 using morphological findings on H\&E slides. Immunohistochemistry was done in 40 cases using Synaptophysin, Chromogranin and Neuron specific enolase.

Results: There were 886 neoplasms diagnosed in GIT of which $53(5.98 \%)$ were NET. The mean age of presentation was 50 years. The male: Female ratio observed is $2: 1$. The most common presenting symptoms were abdominal pain followed by loss of appetite and weight. Carcinoid syndrome was seen in 2/53 (3.8\%) patients. The most common site involved was Stomach followed by duodenum and ileum. NET Grade 1 was seen in 22 cases, NET Grade 2 was seen in 9cases, NET Grade3 was seen 4 cases and mixed adenocarcinoma and neuroendocrine carcinoma (MANEC) was seen in 18 cases. Metastasis to liver was seen in 3 cases Most of the NET tumors expressed the IHC markers, 95\% were positive for NSE, $87.5 \%$ were positive for Synaptophysin and $82.5 \%$ cases were positive for Chromogranin

Conclusion: Neuroendocrine tumors (NETs) are uncommon malignancies of GIT. Stomach was the most common anatomical site. NET grade 1 was the most common histological subtype. IHC markers NSE, Synaptophysin and chromogranin can be used in diagnosis of NETs
\end{abstract}

Keywords: Neuroendocrine tumors, Gastrointestinal tract, Histomorphology, Immunohistochemistry, Grading.

\section{Introduction}

Neuroendocrine neoplasms are derived predominately from enterochromaffin or Kulchitsky's cells and have diverse pathologic findings that typically correspond to the site of origin and hormone-secreting ability. ${ }^{1}$ These tumors are found in the lung, ovary, and biliary and gastrointestinal tracts. The estimated prevalence of neuroendocrine tumors (NET) is 1 to 2 cases per 100,000 people, of which gastrointestinal (GI) tract is the most common site. ${ }^{2}$ The incidence of GI NET is around $67.5 \%$ amongst all NET. ${ }^{3}$ There are no epidemiological data regarding the incidence or prevalence of NET of GIT available from Indian literature. Among the various neuroendocrine tumors (NETs) in the gastrointestinal tract, the small intestine is the commonest site of occurrence and carcinoid tumor is the common pathological type. ${ }^{4-6}$ Neuroendocrine tumors include a spectrum of lesions that encompasses everything from carcinoid tumors (NET Grade1), Atypical carcinoid / Intermediate neuroendocrine tumor (NET Grade 2) and neuroendocrine carcinomas (NET Grade3) Majority of these Neuroendocrine tumors have an indolent course. Some are diagnosed incidentally while few have disseminated disease and may present as metastatic disease. Histological analysis often fails to distinguish the aggressive and metastatic potential of the tumor. Immunohistochemical stains such as chromogranins, synaptophysin and neuron specific enolase are used to identify neuroendocrine tumors and $\mathrm{Ki} 67$ is used in grading the tumors into NET Grade 1, NET Grade 2, and NET Grade $3 .{ }^{5}$ In this study we have analyzed the clinicopathological, histomorphological and immunohistochemical study of neuroendocrine tumors of gastrointestinal tract.

\section{Aims and Objectives}

1. To evaluate the anatomical distribution of Neuroendocrine tumors of GIT.

2. To study the Histomorphological types of Neuroendocrine tumors of GI

3. To evaluate the expression of Immunohistochemical markers in NET.

\section{Materials and Methods}

This was a cross sectional descriptive study that included all specimens of neuroendocrine tumors received from the Department of Surgery and Department of digestive health diseases during the period from September 2008 to September 2012 were included. A detailed history regarding the age, sex, 
clinical symptoms, site, were collected from the medical records in all cases.

The specimens were received and fixed in $10 \%$ neutral formalin for $18-24$ hours and was sampled as per standard guidelines and submitted for processing. The tissues were processed in various grades of alcohol and xylol using automated histokinette and was stained for Haematoxylin and Eosin. The tumors were classified based on WHO classification 2010 using morphological findings on $\mathrm{H} \& \mathrm{E}$ slides.

Immunohistochemistry was done only in 40 cases. IHC could not be done in those cases where blocks could not be retrieved or where tissues had been exhausted. The immunohistochemical stains used were Synaptophysin, Chromogranin, and Neuron specific enolase.

\section{Immunohistochemical stains}

The following Immunohistochemical antibodies were used from the Biogenex laboratories.

1. Neuron specific enolase (NSE) used was Mouse monoclonal (MIG-N3).

2. Chromogranin A, Mouse Monoclonal (LK2H10), IgG1, Kappa

3. Synaptophysin, Mouse Snp 88, IgG3, Kappa.

Sections for Immunohistochemistry were taken on Slides coated with chrome alum. Sections were subjected to antigen retrieval using pressure cooker technique using citrate retrieval solution $(\mathrm{pH} \mathrm{6)}$ and then treated by Horse Radish Peroxidase (HRP) polymer techniques.

Synaptophysin showing cytoplasmic or membranous granular-brown staining was considered Positive

Chromogranin and NSE: showing cytoplasmic brown staining was considered Positive
Statistical analysis was done based on Microsoft excel version 2010. The frequency of all variables was analyzed.

\section{Observation and Results}

There were 886 neoplasm diagnosed in GIT totally during the five year period of study (September 2008 September 2012) out of which $53(5.98 \%)$ were neuroendocrine tumors. The age of the patients ranges from 25- 71 years with the mean age of presentation of 50 years. .

The most common presenting symptoms were abdominal pain followed by loss of appetite and loss of weight and vomiting (chart-1). Carcinoid syndrome characterized by the complex of flushing, diarrhea, abdominal pain, and occasional asthma was seen in $2 / 53(3.8 \%)$ patients.

\section{Chart 1: Clinical presentation of neuroendocrine} tumors

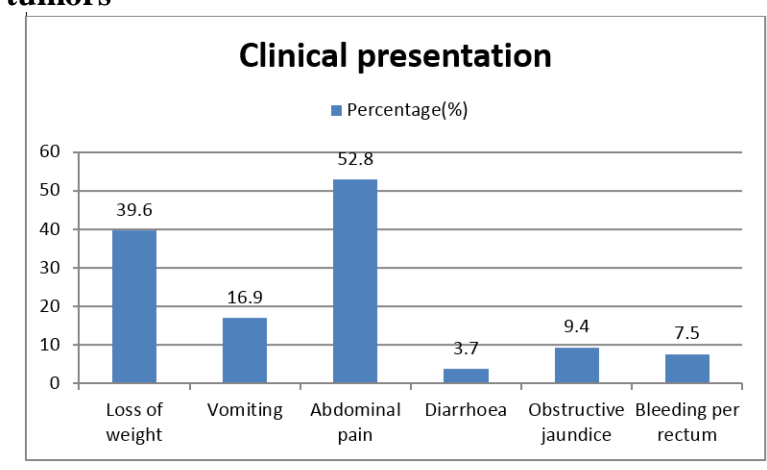

Out of 53 specimens 45 were biopsy specimens and 8 were resection specimens. The most common site involved was Stomach followed by duodenum and ileum, tabulated in Table 1.

Table 1: Anatomical site and histomorphological grading of neuroendocrine tumors

\begin{tabular}{|l|c|c|c|c|c|}
\hline \multicolumn{1}{|c|}{ Site } & NET GI & NET G2 & NEC & MANEC & $\begin{array}{c}\text { Total no of } \\
\text { cases n(\%) }\end{array}$ \\
\hline Oesophagus & 1 & 0 & 0 & 0 & $1(1.9)$ \\
\hline Stomach & 8 & 6 & 1 & 9 & $24(45.3)$ \\
\hline Small intestine & 10 & 2 & 1 & 5 & $18(34)$ \\
\hline Appendix & 1 & 0 & 0 & 0 & $1(1.9)$ \\
\hline Colon & 0 & 0 & 2 & 3 & $5(9.4)$ \\
\hline Rectum & 2 & 1 & 0 & 1 & $4(7.5)$ \\
\hline Total $\mathrm{n}(\%)$ & $22(41.5)$ & $9(17)$ & $4(7.5)$ & $18(34)$ & $53(100)$ \\
\hline
\end{tabular}

On histopathological examination Neuroendocrine tumor G1 was seen in 22 cases, neuroendocrine tumor G2 was seen in 9cases, NEC was seen 4 cases and MANEC was seen in 18 cases (Fig. 1). The distribution of different types of NET at various sites in GIT is tabulated in Table 1. Metastasis was seen in 3 cases and all cases were NET G3 and all showed metastasis to liver. 


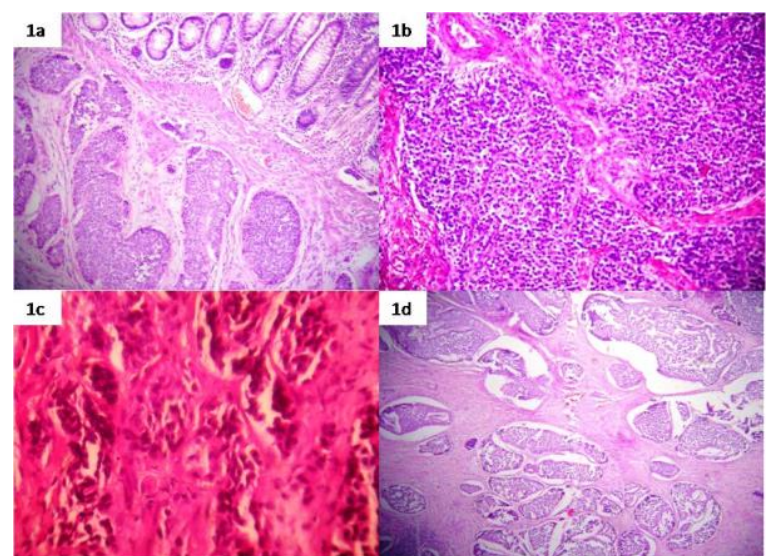

Fig. 1: Histomorphology of neuroendocrine tumors of GIT; (a): Neuroendocrine tumor G1 (H\&E, 100X); (b): Neuroendocrine tumor G2 (H\&E, 400X); (c): Neuroendocrine carcinoma G3 (H\&E, 400X); (d): MANEC (H\&E, 100X)

IHC was done in 40 cases out of which 38 cases (95\%) were positive for NSE, 35 cases $(87.5 \%)$ were positive for Synaptophysin and $33(82.5 \%)$ cases were positive for Chromogranin (Fig. 2). NSE was positive in more cases than Synaptophysin followed by Chromogranin.

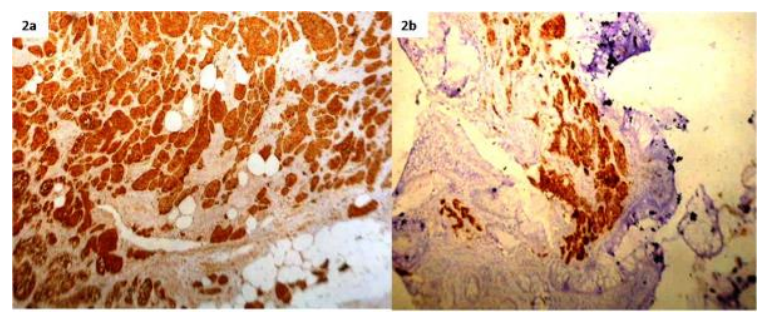

Fig. 2: Immunohistochemical markers showing positivity in Neuroendocrine tumors; (a): Synaptophysin (IHC, 400X); (b): Chromogranin (IHC, 100X)

\section{Discussion}

Gastrointestinal NETs are rare malignant tumors. Due to improved diagnostic and therapeutic modalities, they have gained attention over last few years. In our study done during September 2008 to September 2012, neuroendocrine tumors contributed $3.7 \%$ of the total Malignancies. According to Oberg $\mathrm{k}$ et al the incidence is 2.5 to 5 per 100000 populations. This indicates the low incidence of these tumors. But According to Oyvind Hauso et al the incidence of neuroendocrine tumors is on the rise. ${ }^{7}$

The mean age at diagnosis of NET of GIT in the present study was 50 years. Rothenstein $\mathrm{J}$ et al, ${ }^{8}$ Bruna Estrozi et $\mathrm{al}^{9}$ and Amarapurkar DN et $\mathrm{al}^{10}$ also was showed the Mean age at diagnosis as 56, 52.8 and 53 respectively which correlated with the present study.. Neuroendocrine tumors are rare in paediatric age group. The youngest person affected in this study was 25 yrs and the oldest one was $71 \mathrm{yrs}$.
Rothenstein J et al study and Amarapurkar DN et al study showed that Males were more commonly involved in Neuroendocrine tumors of GIT as seen in our study. ${ }^{8,10}$

The most common site in GIT in our study is the stomach. Our study correlated with Amarpurkar et al ${ }^{10}$ study conducted in India with stomach being the common site. Amarpurkar et al study also showed that next common site was small intestine followed by Rectum and colon. A study in Brazil by Bruna Estrozi et al also showed that the most common site was Stomach followed by small intestine. ${ }^{9}$ Whereas in a study by Borislav et al $^{11}$ small intestine is the most common site with $52.6 \%$ followed by rectum and colon.

Hodgson et $\mathrm{al}^{12}$ in 2005 have shown statistically significant eight or nine fold increase in the incidence of gastric Neuroendocrine tumors. Modlin et $\mathrm{al}^{13}$ have shown significant increase in incidence of gastric neuroendocrine tumors from 2.4 to $8.7 \%$. A study from India by Hegde et al has also shown rising incidence of gastric NETs as compared to the past. ${ }^{2}$ This increase can be attributed to widespread use of proton pump inhibitors or increased endoscopic surveillance with expertise in reporting gastric biopsies. ${ }^{2}$ The most common presenting symptom in our study was Abdominal pain which was comparable with Amarapurkar et al study. ${ }^{10}$ Other presenting symptoms were vomiting, loss of weight and appetite, diarrhea and bleeding per rectum.

In our study NET G1 was the most common histologic type followed by NET G2 and Neuroendocrine carcinomas respectively which correlates with literature where Rothenstein $\mathrm{J}$ et al and Amarapurkar et al also found NET G1 as the most common tumor. ${ }^{14,10}$

Carcinoid syndrome is frequently discussed in relation to carcinoid tumors. However, the complex of flushing, diarrhoea, abdominal pain, and occasional asthma or right-sided valvular problems is actually uncommon. Ito $\mathrm{T}$ et al and Soga $\mathrm{J}$ et al study also show that less than $10 \%(1.7 \%-8.4 \%)$ of neuroendocrine tumors exhibit some of these symptoms. ${ }^{15,16}$ In a study conducted by Warrell et al. (2003) Carcinoid syndrome occured in $10 \%$ of the cases. ${ }^{17}$ In our study there were only two cases $(3.8 \%)$ of total as comparable with Amarpurkar DN et al study conducted in India with $4.1 \%$. Both the cases were carcinoid tumors (NETG1) of appendix that presented with Carcinoid syndrome.

Matsui K et al and Okita NT et al showed malignant tumours with mixed glandular and neuroendocrine characteristics; with at least $30 \%$ of one component was seen in $34 \%$ of cases. They should be diagnosed because treatment depends on both the components. ${ }^{18,19}$

According to Matsui $\mathrm{K}$ et $\mathrm{a}^{18}$ and Okita NT et $\mathrm{al}^{19}$ neuroendocrine carcinoma is a rare tumor with highly malignant biological behavior exhibiting aggressive growth that leads to vascular invasion, distant 
metastasis and extremely poor prognosis. According to Rothenstein $\mathrm{J}$ et al ${ }^{14}$ metastasis was observed in $53 \%$ of the cases. In a study by Amarapurkar et $\mathrm{al}^{10}$ Metastasis was seen in $18.9 \%$ of cases but our study showed Metastasis in only $5.7 \%$ ( 3 cases) of the cases and all the three cases were NET G3 which showed metastasis to liver. Two of which were from stomach and one case was from small intestine. Immunohistochemical studies were used to confirm the diagnosis of neuroendocrinetumors. Our study also showed that most of the NET tumors expressed the IHC markers NSE and Synaptophysin compared to chromogranin. Anna Fen-Yau Li et al also found that NSE and Synaptophysin to be the most useful markers in confirming Neuroendocrine tumors. ${ }^{20}$

\section{Conclusion}

Neuroendocrine tumors (NETs) are uncommon malignancies of GIT. Stomach was the most common anatomical site. NET Grade 1 was the most common histological type. IHC markers NSE, Synaptophysin and Chromogranin can be used in diagnosis of NETs.

Funding: No funding sources.

Conflict of interest: None declared.

\section{References}

1. Ramage JK, Davies AH, Ardill J, et al. "Guidelines for the management of gastroenteropancreatic neuroendocrine (including carcinoid) tumours". Gut. 54 Suppl 4 /gut.2004.

2. Hegde V, Mohandas KM, Ramadwar M, Shukla P, Mehta S. Gastric carcinoids - a changing trend. Indian $J$ Gastroenterol 2003;22:209-11.

3. Sippel RS, Chen H. Carcinoid tumors. Surg Oncol Clin N Am 2006;15:463-78.

4. Maggard MA, O'Connell JB, Ko CY. Updated populationbased review of carcinoid tumors. Ann Surg 2004;240:11722.

5. Modlin, I. M.; Shapiro, M. D.; Kidd, M. "Siegfried oberndorfer: Origins and perspectives of carcinoid tumors". Human Pathol 2004;35(12):1440-51.

6. Radhakrishnan S, Subramoniam S. Colerectal carcinoids in South India. Trop Geogr Med 1979;31:63-7.

7. OyvindHauso, MD,Bjorn I. Gustafsson, $\mathrm{MD}, \mathrm{PhD}$. Neuroendocrine Tumor Epidemiology American Cancer Society2008;2655-2664

8. Rothenstein J, Cleary SP, Pond GR, Dale D, Gallinger S, Moore MJ, Brierley J, Siu LL. Neuroendocrine tumors of the gastrointestinal tract: a decade of experience at the
Princess Margaret Hospital. Am J Clin Oncol 2008;31(1):64-70.

9. BrunaEstrozi, Carlos E. Bacchi Neuroendocrine tumors involving the gastroenteropancreatic tract: a clinicopathological evaluation of 773 cases. Clinics 2011;66(10):1671-75.

10. Amarapurkar DN, Juneja MP, Patel ND, Amarapurkar $\mathrm{AD}$, Amarapurkar PD. A retrospective clinico-pathological analysis of neuroendocrine tumors of the gastrointestinal tract. Trop Gastroenterol 2010;31(2):101-4.

11. Borislav A Alexiev, Cinthia B Drachenberg, John C Papadimitriou, Endocrine tumors of the gastrointestinal tract and pancreas: grading, tumor size and proliferation index do not predict malignant behaviour Diagnostic Pathology 2007; $1597: 2-8$

12. Hodgson N, Koniaris LG, Livingstone AS, Franceschi D. Gastric carcinoids: a temporal increase with proton pump introduction. Surg Endosc 2005:19:1610-2.

13. Modlin IM, Lye KD, Kidd M. A 50-year analysis of 562 gastric carcinoids: small tumor or larger problem? Am J Gastroenterol 2004;99:23-32.

14. Rothenstein J, Cleary SP, Pond GR, Dale D, Gallinger S, Moore MJ,et al. Neuroendocrine tumors of the gastrointestinal tract: a decade of experience at the Princess Margaret Hospital. Am J Clin Oncol 2008;31(1):64-70.

15. Ito T, Tanaka M, Sasano H, Osamura YR, Sasaki I, Kimura $\mathrm{W}$, et al Preliminary results of a Japanese nationwide survey of neuroendocrine gastrointestinal tumors. J Gastroenterol 2007;42:497-500.

16. Soga J, Yakuwa Y, Osaka M. Carcinoid syndrome: a statistical evaluation of 748 reported cases. J Exp Clin Cancer Res 1999;18:133-41.

17. Warrell et al. (2003). Oxford Textbook of Medicine (4th ed.). Oxford University Press

18. Matsui K, Kitagawa M, Miwa A, Kuroda Y, Tsuji M. Small cell carcinoma of the stomach: a clinicopathologic study of 17 cases. Am J Gastroenterol 1991;86:1167-75.

19. Okita NT, Kato K, Takahari D, Hirashima Y, Nakajima TE, Matsubara J, Hamaguchi T, Yamada Y, Shimada Y, and Taniguchi H, Shirao K. Neuroendocrine tumors of the stomach: chemotherapy with cisplatin plus irinotecan is effective for gastric poorly-differentiated neuroendocrine carcinoma. Gastric Cancer 2011;14:161-65.

20. Anna Fen-Yau Li, Alice Chia-Heng Li .Small cell carcinomas in gastrointestinal tract: immunohistochemical and clinicopathological features. J Clin Pathol 2010;63:62025.

How to cite this article: J. Jeya, R. Narmadhar, V. Rajalakshmi, S. Kalaivani. Clinicopathological profile of neuroendocrine tumors of gastrointestinal tract. $J$ Diagn Pathol Oncol 2018;3(4):286-89. 